\title{
Reconstructing Rooms Using Photon Echoes: A Plane based model and reconstruction algorithm for looking around the corner
}

\author{
Adithya Kumar Pediredla \\ Rice University \\ Houston, Texas, USA \\ adithya.k.pediredla@rice.edu
}

\author{
Mauro Buttafava and Alberto Tosi \\ Dipartimento di Elettronica, Informazione e Bioingegneria \\ Politecnico di Milano, Milan, Italy \\ mauro.buttafava@polimi.it and alberto.tosi@polimi.it
}

\author{
Oliver Cossairt \\ Northwestern University \\ Evanston, Illinois, USA \\ olliedeecs.northwestern.edu
}

\author{
Ashok Veeraraghavan \\ Rice University \\ Houston, Texas, USA \\ vashokerice.edu
}

\begin{abstract}
Can we reconstruct the entire internal shape of a room if all we can directly observe is a small portion of one internal wall, presumably through a window in the room? While conventional wisdom may indicate that this is not possible, motivated by recent work on 'looking around corners', we show that one can exploit light echoes to reconstruct the internal shape of hidden rooms.

Existing techniques for looking around the corner using transient images model the hidden volume using voxels and try to explain the captured transient response as the sum of the transient responses obtained from individual voxels. Such a technique inherently suffers from challenges with regards to low signal to background ratios (SBR) and has difficulty scaling to larger volumes. In contrast, in this paper, we argue for using a plane-based model for the hidden surfaces. We demonstrate that such a plane-based model results in much higher SBR while simultaneously being amenable to larger spatial scales. We build an experimental prototype composed of a pulsed laser source and a singlephoton avalanche detector (SPAD) that can achieve a time resolution of about 30ps and demonstrate high-fidelity reconstructions both of individual planes in a hidden volume and for reconstructing entire polygonal rooms composed of multiple planar walls.
\end{abstract}

\section{Introduction}

Over the last few years, researchers have demonstrated that using information contained in transient light echoes, one can reconstruct objects that are outside the line of sight $[23,37,16]$. Unfortunately, progress in 'looking around the corner' (LATC) has been slow since the early results by Velten et al. [37] and Kirmani et al. [23, 24]. Current techniques still rely on a voxel based representation to model

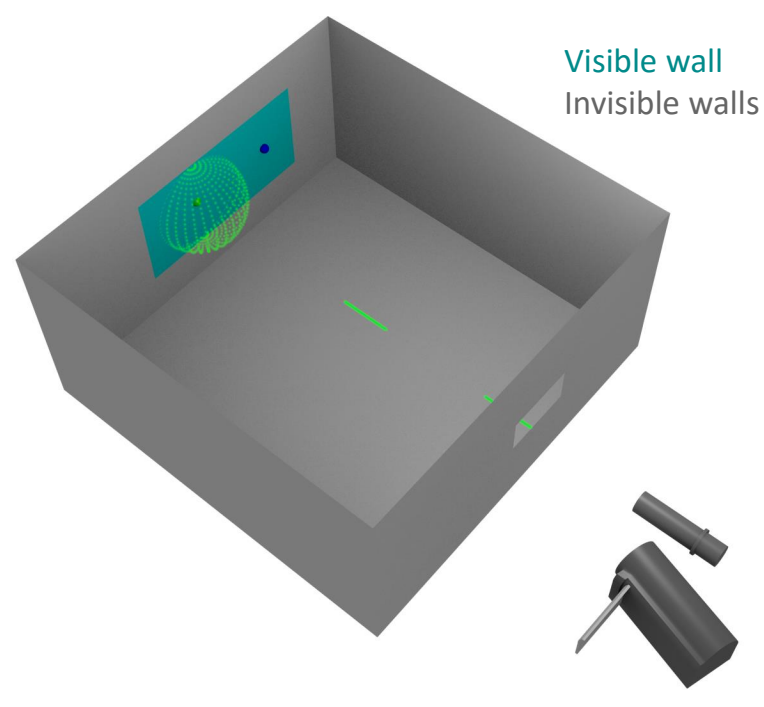

Figure 1. Can we reconstruct the shape of a hidden closed room with a small peephole? We will show an experimental set up, built with a transient camera and a pico-second laser, that can infer the shape of a hidden room. The transient camera consists of single SPAD detector (single-photon avalanche diode) with 30 ps jitter. The pico-second laser has a jitter comparable to that a SPAD, and emits pulses at 530-570 nm wavelength. Coherence lengths at this bandwidth are too low to do interferometric measurements. Instead, we rely on the arrival times of photon echoes in our algorithm.

the hidden scene and attempt to reconstruct the hidden volume by expressing the observed transient response as a sum of the transients produced by individual voxels that make up the hidden volume. With such an approach, there are three main challenges that currently limit progress in practical and realistic application scenarios. 


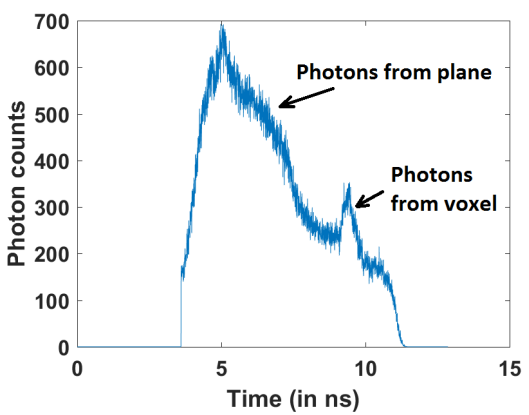

(a) Low signal to background ratio

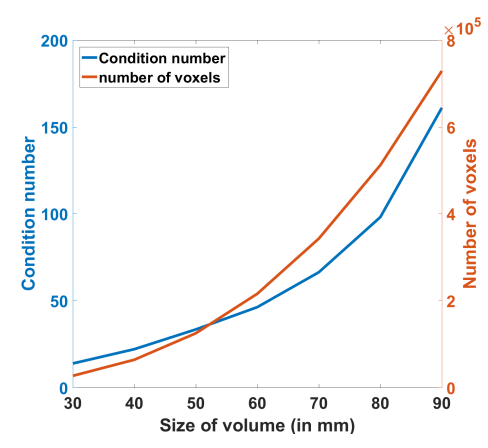

(b) Ill conditioning for large volumes

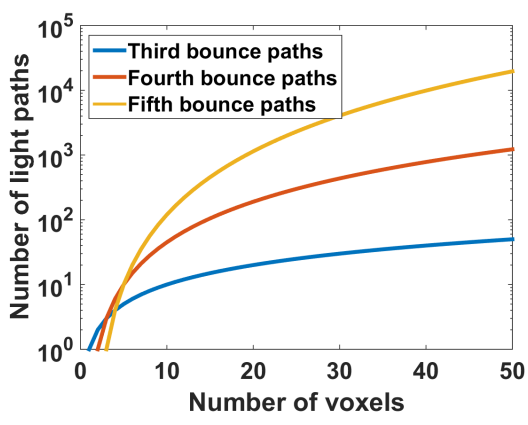

(c) Higher-order bounces

Figure 2. Challenges with current voxel-based reconstructions: (a) Third bounce photons from an individual voxel form a very small fraction of the returning third bounce photons, which by themselves are small in number. The plot shows the captured transient data when the scene consists of a large plane and a small voxel, clearly showing higher SBR for the plane. (b) voxel-based reconstructions suffer from ill-conditioning as the volume being reconstructed increases as demonstrated by rapidly worsening condition number. (c) The light paths that correspond to higher order bounces rapidly overwhelm the third bounce light paths as the number of voxels increase.

1. Low Signal to Background Ratio (SBR): LATC using transient light echoes relies on the photons that bounced three times. The vast majority of the photons captured are direct reflection photons or photons that have otherwise encountered multiple internal reflections and therefore, act as a large background bias in which the third bounce photon signal is buried. This low SBR is further exacerbated by the use of a voxelbased modeling approach, wherein the photons that are scattered from a small hidden voxel only form a minuscule fraction of the already small number of third bounce photons (refer Figure 2(a)).

2. Scaling to Larger Hidden Volumes: A voxel-based approach inherently means that the number of voxels to be reconstructed increases cubically with the size of the hidden scene. Consequently, the reconstruction problem becomes exceedingly ill-conditioned as the size of the hidden volume increases as shown in Figure 2(b)).

3. Higher-order bounces: While the direct bounce photons can be potentially time-gated (as is the case in our experimental data), the higher-order bounces remain un-modeled and contribute as a source of noise and background. A voxel-based approach to model these higher-order bounces is intractable as can be seen from the fact that even just the number of fourth bounce light-paths increase quadratically (refer Figure 2(c)) in the number of voxels, which as mentioned earlier increases cubically with the size of hidden volume.

As a consequence of these three reasons, existing reconstruction results for LATC typically show a two or three discrete, compact objects placed far away from each other to limit inter-reflections. Our goal in this paper is to consider the toy problem of reconstructing a hidden room and show that a plane-based model for transient light echoes has the potential to overcome some of these above-mentioned limitations.

Plane-based representation: Motivated by the fact that in many problems in computer vision, including stereo, multi-view stereo [12], RGB-D [22], and structure from motion [41] a plane-based model has been shown to outperform voxel-based representations, especially when encountering man-made scenes, urban landscapes and indoor environments we explore the utility of such a representation for LATC. Firstly, such a plane-based representation results in a higher SBR just by virtue of the fact that there are typically several large planar structures in many environments, and the number of photons reflected from these large planar structures can now be modeled together as opposed to being divided among the many hundreds or thousands of voxels that make up this plane. Secondly, such plane-based models are eminently more suitable to scale to larger scenes since we are only modeling the hidden surface, rather than the entire hidden volume. These two facts together result in much better conditioning for the inverse problem when compared to the voxel-based representation. Finally, a plane-based model is a much more compact representation (in terms of the number of planes that make up a hidden scene), and this compactness may provide us with opportunities to directly model and account for the higher-order bounces (not yet demonstrated in this paper).

Contributions: In this paper, we demonstrate that a plane-based representation is a promising approach for LATC by focusing on the following toy-problem as a motivating example. Can we reconstruct the entire internal shape of a room if all we can directly observe is a small portion of one internal wall, presumably through a window in the room as shown in Figure 1? The key technical contributions of this paper are the following:

- Plane-based Representation: We propose a plane- 
based representation for looking around the corner using transient light echoes and develop a dictionary based algorithm for reconstructing a hidden surface as a linear superposition of planes.

- Symmetries: We show that there exist several symmetries in the transient light echoes of a plane and demonstrate practical techniques to avoid these symmetries and ensure reliable unique reconstruction.

- Analysis: We perform a systematic analysis of the effect of various parameters such as the number of virtual sources, the time resolution of transients, the SBR, and the size of visible surface on the reconstruction error.

- Real Experimental Prototype: We build an experimental platform composed of a pulsed laser, a SPAD sensor and a time-correlated single-photon counting module (TCSPC) and demonstrate state-of-the-art results in reconstructing hidden scenes including rooms.

Limitations: While a plane-based representation allows us to outerperform previous reuslts using voxel-based representations, the proposed method for LATC still suffers from many limitations. Firstly, the higher-order bounces have not yet been incorporated into our model and therefore remain a source of noise and background in all our experiments, potentially limiting reconstruction performance. Secondly, our entire approach assumes that the bidirectional reflectance distribution function (BRDF) of the hidden subject/room is lambertian and when this assumption is violated, one will have to make appropriate changes to the model to accomodate varying reflectance of the hidden surfaces. Our actual experimental prototype also introduces a couple of important limitations. The overall time jitter of the system (SPAD detector, TCSPC module, pulsed laser source and broadening due to wall surface reflection) is around $100 \mathrm{ps}$. This time jitter limits the accuracy of our reconstruction. One can employ more expensive sensors with lower jitter to increase the reconstruction accuracy as shown in our simulations. In addition, we use a supercontinuum laser source with pulse repetition rate of $77.78 \mathrm{MHz}$. Hence, the SPAD measurements phase wrap after $12.86 \mathrm{~ns}$ or around $3.86 \mathrm{~m}$. If the hidden volume is larger than $3.86 / 2 \approx 1.9$ meters, this would result in inconsistency due to phase wrapping. So all our real scenes were smaller than this limit. In practice, if we needed to accomodate larger scenes, then the pulse repetition rate of the laser source should be reduced, perhaps by using a pulse-picker.

\section{Related work}

\subsection{Transient imaging}

Nils Abramson [1] might have been the first to capture light-in-flight (or transient image) with holography. Around 30 years later, Velten et al. [36, 38] designed a system based on streak camera and femtosecond laser to image the transient interaction of light with matter. Streak camera sacri- fices spatial resolution for temporal resolution. Hence, Velten et al. used scanning mirrors to acquire a $2 \mathrm{D}$ spatiotemporal light-in-flight image. Gao et al. [13] extended Velten et al.'s technique to capture transient images of dynamic, non-repeatable events by combining compressed sensing techniques with streak camera based light-in-flight imaging. As streak cameras are very expensive, Heide et al. [17] used inexpensive photonic mixer devices (PMD) to capture transient images by incorporating prior knowledge about the light/object interactions in an optimization framework. Lin et al. [28] identified Heide et al.'s approach as one of capturing Fourier transform of the transient image, and proposed a framework to decrease the computational and memory cost of the reconstruction algorithm. Kadambi et al. [19] also captured transient images independently, in the same year as Heide et al., with PMDs by employing M-sequences as coded-illumination. Kadambi et al. deconvolve the images captured by PMD to get the transient response. Tadano et al. [34] increased the temporal resolution of the Kadambi et al.'s technique by employing a light source array. Tadano et al. [33] also built a depth selective camera by using M-sequences as coded illumination. Gariepy et al. [14] were the first to use SPADs to capture transient images directly at 67 ps temporal resolution. The spatial resolution of the camera they used is $32 \times 32$. The transient imagers like SPAD were also redesigned to capture photometric and geometric information from just one photon [25, 32], or image without the need for optical elements [24].

\subsection{Looking around the corners}

To our knowledge, Kirmani et al. [23] first theorized the ability to look around the corners using $5 \mathrm{D}$ space-timeimpulse-response (STIR) of the scene. They showed results on reconstructing hidden patches. Velten et al. [37] demonstrated real experiments for looking around corners using third-bounce reflections and elliptic-back projection technique. Gupta et al. [16], using the same set-up as Velten et al., modeled the forward problem as a linear system, and studied various inverse algorithms for reconstructing hidden shapes. Heide et al. [18] used low-cost PMD sensors, and incorporated sparsity priors on the depth and volume in an optimization framework to reconstruct objects around the corner. Buttafava et al. [5] used single pixel SPADs with a temporal resolution of $30 \mathrm{ps}$. They captured a 3D STIR by scanning the wall with a $515 \mathrm{~nm}$ wavelength mode-locked pulsed laser source, running at $55 \mathrm{MHz}$. We have used a similar transient camera system whose details are given in hardware set up section. Tsai et al. [35] used the information from the first bounce photons alone to reconstruct object normals around the corner. Kadambi et al. [20] derived resolution bounds with the help of array signal processing techniques, and Pediredla et al. [30] derived the bounds using a linear systems approach. Using the wave nature of the light, Katz et al. [21] proposed a transmission matrix based approach which Rangarajan et al. [31] extended to 


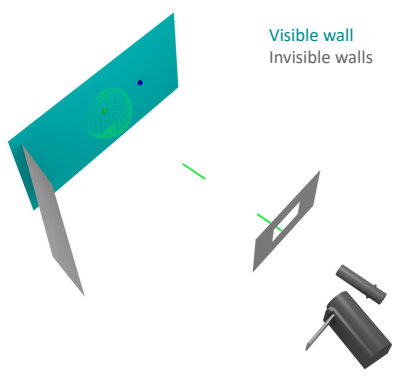

(a) typical scene

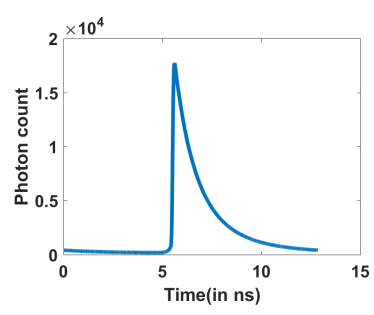

(b) transient response
Figure 3. Forward model: We use a Monte-Carlo based computational model to solve for the transient response of invisible plane. (a) Shows a typical scene. The geometry consists of a visible wall (cyan color in the throughout the paper) and an invisible wall (gray color in the throughout of the paper). The pulsed laser source emits photons that hit the visible wall at a location referred as a virtual source (VS) and marked as a green dot in the scene which emits photons isotropically. Some of these photons hit the invisible wall, and return to the visible wall. The detector is focused on the wall on a location referred as virtual detector (VD) and marked with a blue dot in the scene. The transient response is defined as the photon flux reaching the virtual detector from virtual source. (b) shows the transient response accounting for the jitter of the hardware we have built in the lab.

holography to solve the looking around corners problem.

The reconstruction problem is also extended to track around the corners by Gariepy et al. [15], who used a SPAD array to track objects beyond the line of sight. The acquisition time is around 3 seconds for each position of the hidden object, and hence, this technique can only track slow moving objects. Recently, Kleint et al. [26], used an ordinary 2D intensity camera, to track the translation and orientation of known objects in real time.

\subsection{Hearing the shape of a room}

Our problem statement is similar to the one addressed by Dokmanic et al. $[10,11]$ in the context of audio. By recording the acoustics echoes of the room with multiple microphones and a loud speaker located at fixed known positions inside the room, Dokmanic et al. proposed to reconstruct the shape of the room. The room is assumed to be made of planes and convex in shape. The two important differences between the Dokmanic et al.'s research from ours is the choice the location of transmitter/receiver and the waves used. Dokmanic et al. placed the sensors inside the room whereas our sensors are outside the room. Dokmanic et al. used sound waves, whereas we chose to solve the problem with optical waves. As the surfaces are highly specular (mirror like) at the wavelength of sound (17 millimeters to 17 meters), the problem of room reconstruction is one of finding the correct echoes in each microphone corresponding to any given wall. Though not very easy, Dokmanic et al. used the rank property of Euclidean distance matrices, popularly used in GPS literature [9], to sort the echoes and reconstruct the room. However, at the wavelength of light (400-700nm), the roughness of the materials is comparable enough to make the surfaces diffuse. Therefore the photon echoes have a smooth transient response and we cannot use the ideas developed by Dokmanic et al. directly.

\section{Reconstructing a plane}

Can we reconstruct the entire internal shape of a room if all we can directly observe is a small portion of one internal wall, presumably through a window in the room? We will address this question by first addressing a simpler problem of reconstructing a single infinitely large plane by observing its transient response. Once we have understood this problem, we will then model rooms as composed of many intersecting planes and extend our method to reconstruct a room - one plane at a time.

\subsection{Forward Model}

We parameterize the planes in $\left(z_{i n t}, \theta, \phi\right)$ coordinates, where $z_{\text {int }}$ is the $z$-intercept of the plane, $\theta$ is the angle between plane normal and the $\mathrm{z}$-axis, $\phi$ is the angle between $x$-axis and the projection of plane normal in the $x y$ plane. Note that this parameterization will result in $z_{\text {int }}= \pm \infty$ for all the planes parallel to the $z$-axis ( or $\theta=\frac{\pi}{2}$ ). In this section, we will only consider planes not parallel to the $z$ axis.

Here, we will derive the transient response of a plane. Figure 3 shows the setup of a typical scene. We will refer to the wall visible from the laser/camera pair as the "visible wall" and the other wall plane as the "invisible wall". The source emits a laser beam that hits the visible wall at a location referred to as virtual source (VS). The detector is focused on a point on the visible wall, referred to as virtual detector (VD). The analytical expression for the photon flux $(\Phi(t))$ received at VD at time $t$ is given by

$$
\Phi(t)=I \int_{\forall \bar{p} \in \mathcal{C}(t)} \frac{\cos \angle\left(\bar{v}_{s}-\bar{p}, N_{i}\right) \cos \angle\left(\bar{p}-\bar{v}_{d}, N_{v}\right)}{\left\|\bar{p}-\bar{v}_{s}\right\|^{2}\left\|\bar{p}-\bar{v}_{d}\right\|^{2}},
$$

where $I$ is the photon flux emitted from VS, $\bar{v}_{s}$ and $\bar{v}_{d}$ are the position vectors of VS and VD, $N_{v}$ and $N_{i}$ are the unit normals of the visible wall and invisible wall. $\mathcal{C}(t)$ is the intersection of the invisible wall with the ellipsoid of revolution, whose focal points are $\bar{v}_{s}$ and $\bar{v}_{d}$ and major axis is $\frac{c t}{2}$, where $c$ is the speed of light.

Unfortunately, the analytical formula for the transient response of a plane does not have a closed form expression. This is because the curve $\mathcal{C}(t)$ is an elliptical ring on the plane. The transient photon flux reaching the camera at time $t$ is an integral of the fluxes contributed by points on this elliptic ring. Even the perimeter of an ellipse is not a tractable integral and does not have a closed form expression [6]. 


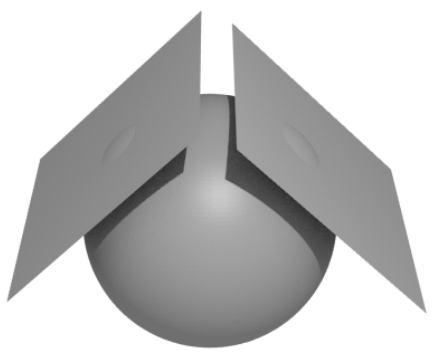

$(a)$

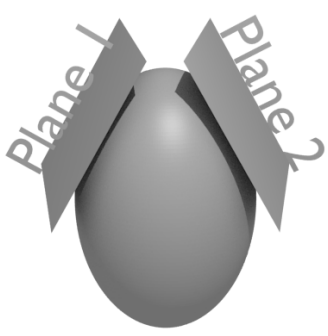

$(b)$

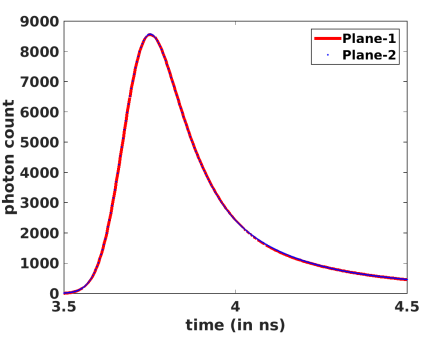

(c)

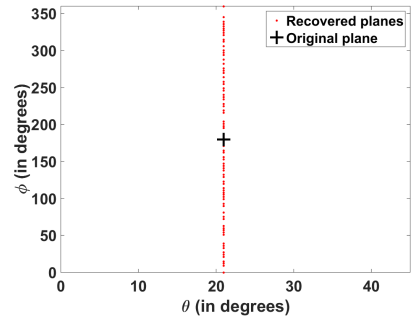

$(d)$

Figure 4. Symmetry for a single transient measurement (a) When VS and VD are chosen to be same point, all the planes that are at a fixed distance have the same transient response and we cannot recover the plane parameters (b) even when the VS and VD are not at the same point, we have a symmetry. All the planes that have same $z_{\text {int }}$ and $\theta$ will have same the same transient response. (c) We show the transient response of two planes that has different $\phi$ but same $z_{i n t}$ and $\theta$. We can notice that the transient responses are identical. (d) For a given plane, we recover the plane parameters using sparse dictionary approach for various realizations of noise and plot the $\theta$ and $\phi$ values of the recovered plane. We can notice that the error in $\theta$ is negligible where as $\phi$ varies arbitrarily over $\left[0,360^{\circ}\right]$.

Hence, it is not possible to have a closed form expression for the transient response of the plane. Therefore, we use a Monte-Carlo based computational model for computing the transient response of the plane, given its geometry.

To estimate the transient response of a room, we generate a million virtual photons uniformly placed on the hemisphere facing the normal of the visible wall and track them. Some of these photons will hit the plane of interest, and the others will be lost into the vacuum. The number of photons that hit the visible wall is a function of the plane normal. For each photon that hits the plane of interest, we compute the travel time and intensity of the flux that reaches the VD by accounting for foreshortening due to the distance and orientation of VD. We compute the transient response by histogramming the photon flux based on the travel time. Note that the transient response corresponds to the time traveled by photons from virtual source to virtual detector and not from the source to the detector. We will show in the experiments section that we can calibrate and remove the bias introduced by the photon travel from source to virtual source and detector to virtual detector.

A sample impulse response of a random plane is shown in Figure 3. We can notice that the response starts after a finite time $t_{0}$, the minimum round trip time from source to the visible plane, and then falls exponentially, reaching close to zero after a few nanoseconds. In practice, the tail has less strength compared to the background level of the transient imaging system. Due to the repetition rate of the laser, the transient response phase wraps after $\sim 12.5 \mathrm{~ns}$. The SPAD system we have used has a temporal jitter higher than the sampling time period. Hence, the transient measurements of the planes are smooth as shown in Figure 3.

\subsection{Symmetry}

Can observing the transient response from any single VS-VD pair result in a unique and accurate estimate of the plane parameters?
The short answer is NO. There is an inherent symmetry in the underlying transient responses that make unique plane identification impossible - multiple planes in the scene will result in the same transient response. As an illustrative example, lets consider the case when VS and VD are co-located, for simplicity at the origin. When a photon travels from the VS, to the object, back to the VD, the roundtrip travel time will depend on the 3D distance from the VS/VD to the point. As a result, there is a locus of scene points along a spherical surface whose distance or time traveled is constant (i.e., the iso-temporal surface). All planes that are equidistant from the origin (i.e, planes with the same perpendicular distance to the origin), are tangential to this spherical iso-temporal surface. The $2 \mathrm{D}$ family of all these planes are symmetric with respect to the origin and will result in the same transient response due to this symmetry. Two such planes and their transient responses are shown in Figure 4(a). To re-state this symmetry explicitly - given a round trip travel time $t_{0}$, we can draw the corresponding iso-temporal surface which is a sphere of radius $r_{0}=c \frac{t_{0}}{2}$. All planes that are tangential to this surface produce the exact same transient response.

Such a symmetry exists not just for the case when VS and VD are co-located, but also for arbitrary VS-VD combinations. For example, consider the case when both VS and $\mathrm{VD}$ are on the z-axis. The resulting iso-temporal surface is a spheroid that is symmetric around $\mathrm{z}$-axis as shown in Figure 4(b). All the planes that make same angle with $z$-axis and have the same $z$-intercept will have the exact same impulse response due the geometric symmetry of the spheroid. This is a little harder to visualize but as shown in Figure 4(c), all of these planes result in the same transient response. Thus, there is now a 1D family of planes that result in exactly the same transient response. Hence, it is not possible to invert the observed transient response and estimate the plane parameters uniquely from the transient responses observed with any single VS-VD pair. In Figure 4(d), we show that it 


\begin{tabular}{|c|ccc|}
\hline $\begin{array}{c}\text { \# virtual } \\
\text { sources }\end{array}$ & 1 & 3 & 10 \\
\hline 4 & 30.52 & 14.43 & 12.43 \\
\hline 8 & 30.21 & 10.45 & 9.73 \\
\hline 16 & 24.30 & 9.33 & 9.90 \\
\hline
\end{tabular}

(a) Reconstruction error in $z_{\text {int }}(\mathrm{mm})$

\begin{tabular}{|c|ccc|}
\hline \# virtual & \multicolumn{3}{|c|}{ SBR } \\
sources & 1 & 3 & 10 \\
\hline 4 & 6.13 & 2.78 & 2.48 \\
\hline 8 & 6.37 & 2.08 & 2.03 \\
\hline 16 & 5.43 & 2.12 & 2.14 \\
\hline
\end{tabular}

(b) Reconstruction error in $\theta^{\circ}$

\begin{tabular}{|c|ccc|}
\hline $\begin{array}{c}\text { \# virtual } \\
\text { sources }\end{array}$ & 1 & 3 & 10 \\
\hline 4 & 27.37 & 3.01 & 1.24 \\
\hline 8 & 17.01 & 1.59 & 0.75 \\
\hline 16 & 18.70 & 1.49 & 0.80 \\
\hline
\end{tabular}

(c) Reconstruction error in $\phi^{\circ}$

Table 1. Simulation: Effect of increasing the number of virtual sources on the reconstruction of a plane

\begin{tabular}{|c|ccc|}
\hline $\begin{array}{c}\text { scan area } \\
(\mathrm{mm} \times \mathrm{mm})\end{array}$ & 1 & 3 & 10 \\
\hline $50 \times 50$ & 51.05 & 33.76 & 25.14 \\
\hline $100 \times 100$ & 45.99 & 20.83 & 14.66 \\
\hline $200 \times 200$ & 30.52 & 14.43 & 12.43 \\
\hline $400 \times 400$ & 19.77 & 9.70 & 9.92 \\
\hline
\end{tabular}

(a) Reconstruction error in $z_{\text {int }}(\mathrm{mm})$

\begin{tabular}{|c|ccc|}
\hline $\begin{array}{c}\text { scan area } \\
(\mathrm{mm} \times \mathrm{mm})\end{array}$ & 1 & 3 & 10 \\
\hline $50 \times 50$ & 10.95 & 7.79 & 6.22 \\
\hline $100 \times 100$ & 9.86 & 4.35 & 2.91 \\
\hline $200 \times 200$ & 6.13 & 2.78 & 2.48 \\
\hline $400 \times 400$ & 3.60 & 1.83 & 1.81 \\
\hline
\end{tabular}

(b) Reconstruction error in $\theta^{\circ}$

\begin{tabular}{|c|ccc|}
\hline $\begin{array}{c}\text { scan area } \\
(\mathrm{mm} \times \mathrm{mm})\end{array}$ & 1 & 3 & 10 \\
\hline $50 \times 50$ & 76.47 & 12.76 & 4.34 \\
\hline $100 \times 100$ & 42.04 & 7.33 & 7.30 \\
\hline $200 \times 200$ & 27.37 & 3.01 & 1.24 \\
\hline $400 \times 400$ & 13.28 & 1.79 & 0.96 \\
\hline
\end{tabular}

(c) Reconstruction error in $\phi^{\circ}$

Table 2. Simulation: Effect of increasing the scan area on the reconstruction of a plane

\begin{tabular}{|c|ccc|}
\hline $\begin{array}{c}\text { temporal } \\
\text { jitter }(\mathrm{ps})\end{array}$ & 1 & 3 & 10 \\
\hline 25 & 15.33 & 12.43 & 12.38 \\
\hline 50 & 20.69 & 12.43 & 12.43 \\
\hline 100 & 30.52 & 14.43 & 12.43 \\
\hline 200 & 45.86 & 15.74 & 12.43 \\
\hline 400 & 71.40 & 23.32 & 12.89 \\
\hline
\end{tabular}

(a) Reconstruction error in $z_{\text {int }}(\mathrm{mm})$

\begin{tabular}{|c|ccc|}
\hline $\begin{array}{c}\text { temporal } \\
\text { jitter (ps) }\end{array}$ & 1 & 3 & 10 \\
\hline 25 & 3.04 & 2.48 & 2.48 \\
\hline 50 & 4.44 & 2.48 & 2.48 \\
\hline 100 & 6.13 & 2.78 & 2.48 \\
\hline 200 & 9.83 & 3.24 & 2.48 \\
\hline 400 & 13.96 & 4.88 & 2.55 \\
\hline
\end{tabular}

(b) Reconstruction error in $\theta^{\circ}$

\begin{tabular}{|c|ccc|}
\hline $\begin{array}{c}\text { temporal } \\
\text { jitter }(\mathrm{ps})\end{array}$ & 1 & 3 & 10 \\
\hline 25 & 8.34 & 1.37 & 1.28 \\
\hline 50 & 14.15 & 1.86 & 1.38 \\
\hline 100 & 27.37 & 3.01 & 1.24 \\
\hline 200 & 46.69 & 6.24 & 1.21 \\
\hline 400 & 78.79 & 13.86 & 2.35 \\
\hline
\end{tabular}

(c) Reconstruction error in $\phi^{\circ}$

Table 3. Simulation: Effect of increasing the temporal jitter of the system on the reconstruction of a plane

is not possible to recover the angle $\phi$ for this example case.

\subsection{Explicit symmetry breaking}

While the symmetry inherent in any single VS-VD pair precludes the possibility of unique identification of a hidden plane, we can fortunately break the symmetry by carefully choosing multiple VS-VD pairs that ensure that the cumulative transient response for each plane is indeed unique. As an example, even when only two VS-VD pairs are chosen carefully, such that they are not collinear, it can be shown that these symmetries can be eliminated ensuring unique and accurate reconstruction of plane parameters. In practice though, we choose several VS-VD combinations for reconstruction since these additional measurements provide robustness to other un-modeled sources of errors such as interreflections and background. Due to practical challenges in the physical implementation, we fixed the location of VD and moved VS with the help of a pair of galvanometric mirrors introduced in the light path of the pulsed laser source. In Figure 5, we show how choosing multiple VS locations, even with a fixed VD location breaks symmetry.

\subsection{Characterization of single plane recovery}

To recover a single hidden plane, we used a dictionarybased approach. Let $D$ be the dictionary of transient responses of various hidden planes. For creating $D$, we first sampled multiple planes uniformly from $z_{\text {int }} \in$ $[200 \mathrm{~mm}, 800 \mathrm{~mm}]$ in steps of $20 \mathrm{~mm}, \theta \in\left[0^{\circ}, 45^{\circ}\right]$ in steps of $3^{\circ}, \phi \in\left[0^{\circ}, 360^{\circ}\right]$ in steps of $3^{\circ}$. We limit the choice of $\theta$ to a max of $45^{\circ}$ as further increase creates planes that go between VS and VD. The transient response of each one of these planes will be a column of the matrix $D$, which is obtained by evaluating the value of the integral in equation 1 at these plane parameters.

Once we have the dictionary $(D)$, and a transient measurement $(\bar{y})$, the hidden plane can be obtained by solving the following optimization problem.

$$
\begin{aligned}
& \arg \min _{\bar{x}}\|\bar{y}-D \bar{x}\|, \\
& \text { subject to } x_{i} \in\{0,1\}, \\
& \sum_{i} x_{i}=n,
\end{aligned}
$$

where $n$ is the number of hidden planes ( $n=1$ for single hidden plane recovery). The above problem is computationally hard and we relax it with the following problem:

$$
\arg \min _{\bar{x}}\|\bar{y}-D \bar{x}\|+\lambda\|\bar{x}\|_{1} .
$$




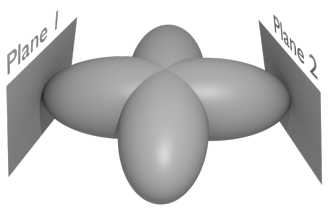

(a)

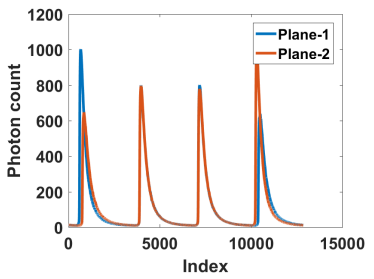

(b)
Figure 5. Symmetry breaking: We break the symmetry by gathering data from more than one VS location. We can notice from the figure that each VS-VD combination has its own symmetry but the entire configuration is not symmetric as the virtual sources are ordered. (b) The concatenated impulse response from all four virtual source locations on both the planes. Notice how the plane responses are different.

The value of $\lambda$ is chosen appropriately so that the sparsity of $\bar{x}$ is $n$. The hidden planes are the ones corresponding to the values of $\bar{x}$ that are non-zero.

For simulation studies, we have to generate the transient response of an arbitrary plane $(\bar{y})$ accounting for noise and background into the ideal transient response. First, the actual observed response is a Poisson process whose rate is given by equation 1 - therefore, the transient response of an arbitrary plane must be a realization of this random process so that the effect of photon counting noise is incorporated. Second, the actual observed response will always contain a background which can be modeled as a Poisson process with a constant rate (the constant depending upon the strength of unmodeled factors including wrap-around due to pulse repetition rate and higher order bounces). We account for both these effects in simulating the transient response $(\bar{y})$ by appropriately sampling from Poisson processes. Other noises such as readout noise are negligible for SPAD.

The reconstruction algorithm is influenced by various experimental parameters, namely signal-to-background ratio, number of virtual sources, the total scan area (where the virtual sources are placed), and temporal jitter of the transient camera. Through simulations, we have systematically evaluated the effect of these parameters in this section. The system we have used in our experiments has an $\mathrm{SBR} \sim 5$, temporal jitter of $100 \mathrm{ps}$. We varied the simulation parameters around these experimental settings and quantified the reconstruction accuracy on randomly generated hundred planes. All these random planes are sampled uniformly from $[200 \mathrm{~mm}, 800 \mathrm{~mm}] \times\left[0^{\circ}, 45^{\circ}\right] \times\left[0^{\circ}, 360^{\circ}\right]$ and the transient response $y$ is computed by appropriately sampling from the Poisson process on the ideal response.

The results of the simulation experiments are in Tables 1-

3. The main conclusions of the simulation experiments are

1. Improving SBR beyond 10 has no significant effect.

2. Increasing number of VS has diminishing returns.

3. Increasing scan area and decreasing temporal jitter improves accuracy, especially at low SBR.

\section{Reconstructing Rooms}

In the previous section, we showed how to estimate plane parameters of an infinite plane from the transient response. Here, we will use those concepts to solve an interesting challenge problem of reconstructing the geometry of a room from the transient response with only one visible surface of the room.

\subsection{Problem setup}

Consider a polygonal room with a small peephole which gives us the ability to see one wall. We will call this wall as a visible wall. There are $\mathrm{n}$ different invisible walls, and the room is made up of all these $(n+1)$ walls. Even on the visible wall, we can observe only a finite area. We want to reconstruct the entire geometry of the room, by which we imply the parameters of all the planes that constitute the room. We want to solve this inference from the transient response of the room obtained by placing virtual sources and detectors on the visible area of the visible wall.

\subsection{Modeling inaccuracies}

One simple method to approach this problem is to model the transient response as a sum of the transient responses that will be observed if each of those individual planes that compose the room were present separately. If such a linear model works, then the problem is solved as we can use linear inversion techniques to solve the problem. Unfortunately, this linear model is inaccurate in the following three ways:

Concave polygons: Assume that the room is a concave polygon from the viewpoint of the visible wall. Hence, there is a point in the room that is not in the direct line of sight of the visible wall. So, only fourth bounce photons are the ones we collect. However, the linear model takes care of only third bounce photons, and hence, we cannot estimate this wall. So, the only kind of rooms we are going to consider are the ones where the room is a convex polygon, which is not a very restrictive assumption as most rooms are convex polygons.

Finite planes: All our planes are assumed to be infinite. However, due to the occlusions caused by other walls, the planes are finite in nature. The finite extent of the walls affects the transient response observed. We will show in Section 5 on how to address this problem.

Higher order bounces: When we consider single planar surface, only third bounce photons reach the detector. When we have multiple surfaces, the fourth, fifth and higher order bounces also reach the detector. These light paths are not modeled in the original planar model, which causes another source of error. We will show in Section 5 on how to handle both the finite plane and higher order bounce errors together. 


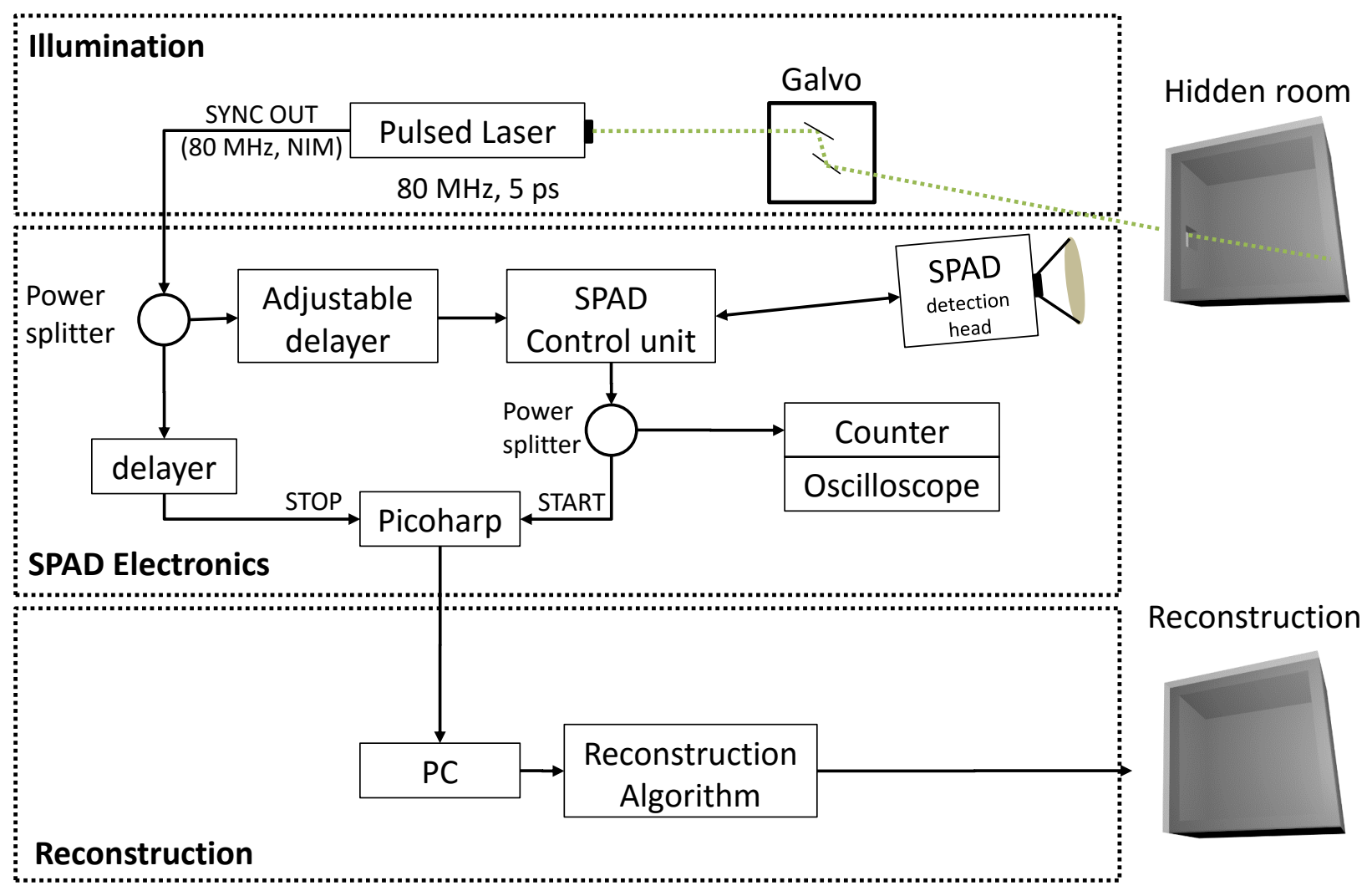

Figure 6. Block diagram of experimental setup: The three core components of our setup are illumination hardware, SPAD electronics, and reconstruction algorithm. The illumination hardware consists of a pulsed laser, which sends periodic pulses of short duration and a galvo to control the position of the beam. The SPAD electronics consists of SPAD to detect a photon and Picoharp to compute the timing of that photon. The data from Picoharp is fed to a computer where the reconstruction algorithm will computationally determine the shape of the room.

\section{Handling non-linearities}

Within the construction of convex polygons, now let us look at how the problems induced by finite planes and interreflections can be handled. We will use a two-step algorithm. First, we will create an initial estimate of the room, then we will refine the initial estimate of the room, by taking into account the finiteness and inter-reflections between the planar surfaces.

\subsection{Initial estimate of the walls}

Consider the case where the virtual source and detector on the visible wall are at least a distance $150 \mathrm{~mm}$ away from the nearest invisible wall. The third bounce photons reach the detector much before the fourth and other higher order bounces. Hence, we can solve the problem using third bounce photons.

\subsection{Refining the estimate of the wall}

When we have finite walls, we have two factors that cause the difference between the transient response of the finite wall to an infinite wall, namely non-linear attenuation factor, and the time extent. The time extent, where the transient response of the wall is non-zero, is smaller for a finite wall, compared to that of an infinite wall. The non-linear attenuation factor comes into picture due to the points that are within the time extent of the finite wall but are not part of the finite wall. We reflect the effect of these parameters on the dictionary and find a more accurate estimate of the walls. Once we obtain a more precise estimate of the shape of the room, we repeat the process till convergence.

\section{Hardware setup}

The block diagram of our setup is shown in Figure 6. The set up can be broken into three main components: (i) picosecond pulsed laser illumination, (ii) single-photon detection and TCSPC electronics and (iii) reconstruction algorithm. The laser source is used to synchronize the entire setup, triggering the SPAD-based detection module and acting as a STOP signal for the time-measurement unit.

\subsection{Illumination}

A picosecond supercontinuum laser (SuperK Extreme, EXW-12, NKT Photonics A/S) is used as illumination 
source. The laser generates light pulses with around $5 \mathrm{ps}$ width at $77.78 \mathrm{MHz}$ repetition rate, with a broad wavelength range (between 400 and $2400 \mathrm{~nm}$ ). We used tunable band pass filter (SuperK Varia, NKT Photonics A/S) to select a wavelength range of 530-570 $\mathrm{nm}$. The output from the filter is injected into an optical fiber and then is collimated with an achromatic aspherical collimator (PAF-X-11-PCA, Thorlabs Inc.). The average output power of the freespace beam reaches $50 \mathrm{~mW}$, and the beam diameter is approximately $3 \mathrm{~mm}$. The position of the virtual source on the wall is controlled using a 2-axis galvanometric mirror (GVS-012, Thorlabs Inc.) able to scan 40 degrees optical angle in both $\mathrm{x}$ and $\mathrm{y}$ directions.

\subsection{Single-photon detection and TCSPC electronics}

We used gated-mode single-photon detection module developed at Politecnico di Milano (Milano, Italy) as transient camera [4]. It is based on a silicon SPAD [8] with a circular active area of $50 \mu \mathrm{m}$ diameter [39]. The module exhibits a detection efficiency around $30 \%$ at $550 \mathrm{~nm}$, a dark count rate lower than 50 counts per second (at $15^{\circ} \mathrm{C}$ temperature) and a temporal response narrower than 35 ps Full-Width at Half Maximum (FWHM). SPAD count-rate saturates at around $8 \times 10^{6}$ counts per second. Hence, to prevent saturation, we exploit the gated-mode operation capability of the detection system, to reject the first bounce photons. The gate duration is set to $6 \mathrm{~ns}$, during which the SPAD can detect photons. The time position of the gate respect to the laser pulse is controlled using an adjustable pico-second delayer, with 50 ns delay range and 10 ps resolution (Microphoton Devices s.r.l.).

Every time a photon is detected, the module sends a NIM (Nuclear Instrument Module) compatible voltage signal to the TCSPC unit. TCSPC technique $[29,2]$ relies on the precise measurement of the time elapsed between each detected photon and the corresponding illumination pulse, building a histogram of photon arrival times to reconstruct the waveform of optical signal under investigation, with picosecond resolution. We used a PicoHarp 300 (PicoQuant $\mathrm{GmbH}$ ) as TCSPC module. The NIM output pulses of the picosecond laser and SPAD module are used as input channels. The bin-width of the PicoHarp 300 is 4 ps, and its maximum conversion rate is around $10^{7}$ conversions per second, well above the SPAD saturation count rate. The resulting histograms are saved to a PC for further data processing. The measured jitter of the overall system is around $100 \mathrm{ps}$ FWHM. This value is the sum of the contributions from the laser source, SPAD module, TCSPC unit and the broadening of the light pulse when reflected by the wall.

\subsection{Reconstruction algorithm}

For calibration, we remove the time bias introduced by SPAD electronics, time travel between source to VS and detector to VD. We first measure the transient response recorded by SPAD when the laser is pointing towards VD.

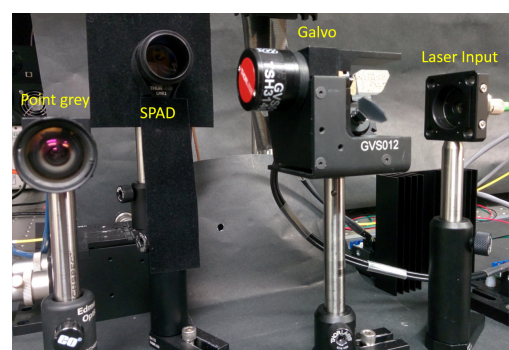

(a) Imaging hardware

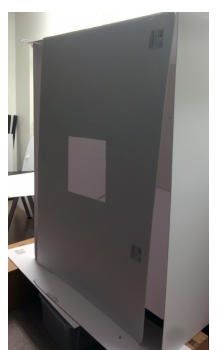

(b) Example room
Figure 7. Hardware setup: (a) The image hardware consists of fiber optic laser input, galvo, and SPAD. We also used a CMOS camera (Point Grey Research Inc.) for calibration purposes. (b) We built the room with white foam boards. Though they are weak and sag on their own weight, foam boards offer the flexibility of reconfiguring the room shapes. The hole in the front foam board is the peephole of our setup.

From peak detection, we find the time that corresponds to the total travel time between the source to the VD, VD to the detector, and other delays in the electronic circuits. We physically measured the distance between the VS and the wall to remove the effect for travel time between source and VD from the above time measurement. To account for the travel time between VS to the source, for every scan position, we also measure the location of the VS from the VD using a calibrated CMOS camera (Point Grey Research Inc.). By summing these two delays, we compute the shift we need to incorporate to each histogram. We shift the histogram measured for each VS position appropriately, and the resulting histograms correspond to the travel time between VS to object to VD. We then use the reconstruction algorithm discussed in Section 5 for measuring plane parameters.

\section{Experiments}

In this section, we systematically evaluated the performance of plane reconstruction and room reconstruction.

\subsection{Single plane}

As mentioned in Section 5, we are interested in the computation of plane parameters of the finite plane and not the finiteness parameters as the finite length can be computed by intersecting the walls of the room. We first analyzed the reconstruction accuracy of a single plane by varying the size, orientation, and distance of the plane. The lab was almost sealed off to avoid stray light reaching the detector. However, there was always some amount of background light reaching the SPAD, also due to interreflections, and resulting SBR was around 4. Table 4 summaries the reconstruction accuracies. The intercept, azimuth, and elevation angles have an approximate error of $50 \mathrm{~mm}, 5^{\circ}$ and $9^{\circ}$ respectively.

Here we also introduce a metric inspired by iterative 


\begin{tabular}{|c|ccc|ccc|ccc|c|}
\hline $\begin{array}{c}\text { Dimensions of the } \\
\text { plane }(\mathrm{mm} \times \mathrm{mm})\end{array}$ & \multicolumn{2}{|c|}{ Ground truth } & \multicolumn{2}{|c|}{ Reconstruction } & \multicolumn{3}{c|}{ Error } & AICP \\
\hline $100 \times \times 2000$ & 570 & 13.3 & 0 & 630 & 18 & 354 & 60 & 4.7 & 6 & 46 \\
\hline $1000 \times 2000$ & 490 & 13.3 & 90 & 540 & 12 & 96 & 50 & 1.3 & 6 & 34 \\
\hline $1000 \times 2000$ & 410 & 13.3 & 180 & 450 & 18 & 186 & 40 & 4.7 & 6 & 46 \\
\hline $1000 \times 2000$ & 500 & 13.3 & 270 & 540 & 18 & 246 & 40 & 4.7 & 24 & 50 \\
\hline $1000 \times 2000$ & 600 & 21.1 & 0 & 600 & 12 & 348 & 0 & 9.1 & 12 & 199 \\
\hline $1000 \times 2000$ & 500 & 21.1 & 90 & 540 & 18 & 96 & 40 & 3.1 & 6 & 79 \\
\hline $1000 \times 2000$ & 300 & 21.1 & 180 & 360 & 18 & 180 & 60 & 3.1 & 0 & 51 \\
\hline $1000 \times 2000$ & 460 & 21.1 & 270 & 510 & 24 & 252 & 50 & 2.9 & 18 & 30 \\
\hline $750 \times 750$ & 600 & 21.1 & 0 & 690 & 30 & 354 & 90 & 8.9 & 6 & 46 \\
\hline $750 \times 750$ & 490 & 21.1 & 90 & 510 & 18 & 102 & 20 & 3.1 & 12 & 74 \\
\hline $750 \times 750$ & 300 & 21.1 & 180 & 330 & 30 & 186 & 30 & 8.9 & 6 & 115 \\
\hline $750 \times 750$ & 470 & 21.1 & 270 & 540 & 24 & 264 & 70 & 2.9 & 6 & 28 \\
\hline Average error & - & - & - & - & - & - & 45.83 & 4.78 & 9 & 66.5 \\
\hline
\end{tabular}

Table 4. Real data (Single plane reconstruction): A single plane is placed at different orientations and positions. We measured the transient photon flux with the SPAD + TCSPC hardware.

closest point algorithm which is used in vision literature to compute the distance between two surfaces [3, 40, 7]. We refer to this metric as Average Iterative Closest Point (AICP) error and define it as the average distance between the points in the original finite plane and the reconstructed plane. AICP is independent of the coordinate system and is one number that encompasses the error in intercept, $\theta, \phi$, and the finiteness of the plane. The average AICP for the planes we have reconstructed is around $7 \mathrm{~cm}$.

\subsection{Rooms}

We constructed rooms using foam boards as shown in Figure 7. Foam boards sag under their weight when placed vertically but offer the ease in reconfiguring room. As the single plane reconstructions do not have a lot of interreflections, we have first placed only three hidden walls, without the roof and the front wall. This configuration has significant inter-reflections compared to a single wall. We have observed an AICP error of $84 \mathrm{~mm}$. Notice the increase in the AICP error compared to the reconstruction of a single wall.

We then proceeded with all five hidden walls that will make a closed room. We show the reconstruction results in Figure 8. The AICP error for the walls of the room is $106 \mathrm{~mm}$. The average wall length is $1.1 \mathrm{~m}$. Hence, the percentage AICP error is $9.18 \%$.

Jaccard index [27] is used to compare the similarity of sets. We extend it to compare the room we have reconstructed with the original room and redefine it to quantify the reconstruction accuracy of the rooms as

$$
J=\frac{\mu(O \cap R)}{\mu(O \cup R)}
$$

Where $O$ is the set of voxels inside the original room, $R$ is the set of voxels inside the reconstructed room $\mu$ is the

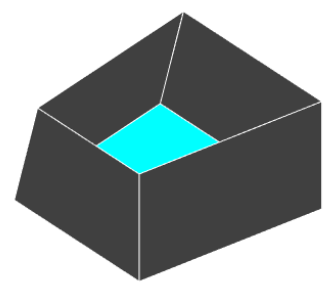

(a) Original room

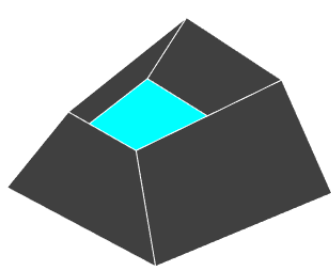

(b) Reconstruction
Figure 8. Real data (Room Reconstruction): (a) A hidden sample room is used for testing the proposed algorithm. The cyan color indicates the visible wall. (b) The resulting wall reconstructions have an average iterative closest point error of $106 \mathrm{~mm}$ $(9.18 \%)$ and Jaccard similarity of 0.63 (higher is better).

cardinality of the set. The Jaccard similarity for both the reconstructed room is 0.63 (higher is better).

\section{Conclusions}

We have proposed a plane-based model for looking around the corner. We showed how symmetries exist in the transient light echoes of a plane and demonstrated practical techniques to break the symmetry. We then performed a systematic analysis of various parameters on the reconstruction of a plane. We used this formulation for the reconstruction of hidden rooms. We built a prototype experimental platform composed of a pulsed laser, SPAD, and time correlated single photon counting module. We used the prototype for reconstruction of single hidden planes and room. Using additional priors such as the right angle between the adjacent walls, the average size of the room can further improve the accuracy of the reconstruction. 


\section{Acknowledgements}

This work was supported in part by NSF CAREER Grant IIS-1453192, DARPA REVEAL grant HR0011-16-C-0028, ONR grant N00014-15-1-2735, ARO grant W911NF-12-10407, and the Big-Data Private-Cloud Research Cyberinfrastructure MRI-award funded by NSF under grant CNS1338099 and by Rice University. The authors would like to acknowledge the help rendered by Prasanna Rangarajan, Tim Gerke, Yicheng Wu, and James Delgado in setting up the hardware.

\section{References}

[1] N. Abramson. Light-in-flight recording by holography. Optics Letters, 3(4):121-123, 1978.

[2] W. Becker. Advanced time-correlated single photon counting techniques, volume 81. Springer Science \& Business Media, 2005.

[3] P. J. Besl and N. D. McKay. Method for registration of 3-d shapes. In Robotics-DL tentative, pages 586-606. International Society for Optics and Photonics, 1992.

[4] M. Buttafava, G. Boso, A. Ruggeri, A. Dalla Mora, and A. Tosi. Time-gated single-photon detection module with $110 \mathrm{ps}$ transition time and up to $80 \mathrm{mhz}$ repetition rate. Review of scientific instruments, 85(8):083114, 2014.

[5] M. Buttafava, J. Zeman, A. Tosi, K. Eliceiri, and A. Velten. Non-lineof-sight imaging using a time-gated single photon avalanche diode. Optics express, 23(16):20997-21011, 2015.

[6] T. R. Chandrupatla and T. J. Osler. The perimeter of an ellipse. Math. Sci, 35:122-131, 2010.

[7] Y. Chen and G. Medioni. Object modelling by registration of multiple range images. Image and vision computing, 10(3):145-155, 1992.

[8] S. Cova, M. Ghioni, A. Lacaita, C. Samori, and F. Zappa. Avalanche photodiodes and quenching circuits for single-photon detection. Applied optics, 35(12):1956-1976, 1996.

[9] J. Dattorro. Convex optimization \& Euclidean distance geometry. Lulu. com, 2010.

[10] I. Dokmanić, Y. M. Lu, and M. Vetterli. Can one hear the shape of a room: The 2-d polygonal case. In 2011 IEEE International Conference on Acoustics, Speech and Signal Processing (ICASSP), pages 321-324. IEEE, 2011.

[11] I. Dokmanić, R. Parhizkar, A. Walther, Y. M. Lu, and M. Vetterli. Acoustic echoes reveal room shape. Proceedings of the National Academy of Sciences, 110(30):12186-12191, 2013.

[12] D. Gallup, J.-M. Frahm, and M. Pollefeys. Piecewise planar and non-planar stereo for urban scene reconstruction. In Computer Vision and Pattern Recognition (CVPR), 2010 IEEE Conference on, pages 1418-1425. IEEE, 2010.

[13] L. Gao, J. Liang, C. Li, and L. V. Wang. Single-shot compressed ultrafast photography at one hundred billion frames per second. $\mathrm{Na}$ ture, 516(7529):74-77, 2014

[14] G. Gariepy, N. Krstajić, R. Henderson, C. Li, R. R. Thomson, G. S. Buller, B. Heshmat, R. Raskar, J. Leach, and D. Faccio. Singlephoton sensitive light-in-fight imaging. Nature communications, 6 , 2015.

[15] G. Gariepy, F. Tonolini, R. Henderson, J. Leach, and D. Faccio. Detection and tracking of moving objects hidden from view. Nature Photonics, 2015.

[16] O. Gupta, T. Willwacher, A. Velten, A. Veeraraghavan, and R. Raskar. Reconstruction of hidden $3 \mathrm{~d}$ shapes using diffuse reflections. Optics express, 20(17):19096-19108, 2012.

[17] F. Heide, M. B. Hullin, J. Gregson, and W. Heidrich. Low-budget transient imaging using photonic mixer devices. ACM Transactions on Graphics (ToG), 32(4):45, 2013.
[18] F. Heide, L. Xiao, W. Heidrich, and M. B. Hullin. Diffuse mirrors: 3d reconstruction from diffuse indirect illumination using inexpensive time-of-flight sensors. In Proceedings of the IEEE Conference on Computer Vision and Pattern Recognition, pages 3222-3229, 2014.

[19] A. Kadambi, R. Whyte, A. Bhandari, L. Streeter, C. Barsi, A. Dorrington, and R. Raskar. Coded time of flight cameras: sparse deconvolution to address multipath interference and recover time profiles. ACM Transactions on Graphics (ToG), 32(6):167, 2013.

[20] A. Kadambi, H. Zhao, B. Shi, and R. Raskar. Occluded imaging with time-of-flight sensors. ACM Transactions on Graphics (TOG), 35(2):15, 2016

[21] O. Katz, E. Small, and Y. Silberberg. Looking around corners and through thin turbid layers in real time with scattered incoherent light. Nature photonics, 6(8):549-553, 2012.

[22] A. Kirmani, A. Colaço, F. N. Wong, and V. K. Goyal. Exploiting sparsity in time-of-flight range acquisition using a single timeresolved sensor. Optics Express, 19(22):21485-21507, 2011.

[23] A. Kirmani, T. Hutchison, J. Davis, and R. Raskar. Looking around the corner using transient imaging. In 2009 IEEE 12th International Conference on Computer Vision, pages 159-166. IEEE, 2009.

[24] A. Kirmani, H. Jeelani, V. Montazerhodjat, and V. K. Goyal. Diffuse imaging: Creating optical images with unfocused time-resolved illumination and sensing. IEEE Signal Processing Letters, 19(1):31-34, 2012.

[25] A. Kirmani, D. Venkatraman, D. Shin, A. Colaço, F. N. Wong, J. H. Shapiro, and V. K. Goyal. First-photon imaging. Science, 343(6166):58-61, 2014.

[26] J. Klein, C. Peters, J. Martín, M. Laurenzis, and M. B. Hullin. Tracking objects outside the line of sight using $2 \mathrm{~d}$ intensity images. Scientific Reports, 6, 2016.

[27] M. Levandowsky and D. Winter. Distance between sets. Nature, 234(5323):34-35, 1971.

[28] J. Lin, Y. Liu, M. B. Hullin, and Q. Dai. Fourier analysis on transient imaging with a multifrequency time-of-flight camera. In Proceedings of the IEEE Conference on Computer Vision and Pattern Recognition, pages 3230-3237, 2014.

[29] D. O'Connor. Time-correlated single photon counting. Academic Press, 2012.

[30] A. K. Pediredla, N. Matsuda, O. Cossairt, and A. Veeraraghavan. linear systems approach to identifying performance bounds in indirect imaging. In To appear in 2017 IEEE International Conference on Acoustics, Speech and Signal Processing (ICASSP). IEEE, 2017.

[31] P. V. Rangarajan and M. P. Christensen. Imaging hidden objects by transforming scattering surfaces into computational holographic sensors. In Computational Optical Sensing and Imaging, pages CTh4B4. Optical Society of America, 2016.

[32] D. Shin, A. Kirmani, V. K. Goyal, and J. H. Shapiro. Photon-efficient computational 3-d and reflectivity imaging with single-photon detectors. IEEE Transactions on Computational Imaging, 1(2):112-125, 2015.

[33] R. Tadano, A. Kumar Pediredla, and A. Veeraraghavan. Depth selective camera: A direct, on-chip, programmable technique for depth selectivity in photography. In Proceedings of the IEEE International Conference on Computer Vision, pages 3595-3603, 2015.

[34] R. Tadano, A. K. Pediredla, K. Mitra, and A. Veeraraghavan. Spatial phase-sweep: Increasing temporal resolution of transient imaging using a light source array. In 2016 IEEE International Conference on Image Processing (ICIP), pages 1564-1568, Sept 2016.

[35] C.-Y. Tsai, K. N. Kutulakos, S. G. Narasimhan, and A. C. Sankaranarayanan. The geometry of first-returning photons for non-line-ofsight imaging. In CVPR, 2017.

[36] A. Velten, E. Lawson, A. Bardagjy, M. Bawendi, and R. Raskar. Slow art with a trillion frames per second camera. In ACM SIGGRAPH 2011 Talks, page 44. ACM, 2011.

[37] A. Velten, T. Willwacher, O. Gupta, A. Veeraraghavan, M. G. Bawendi, and R. Raskar. Recovering three-dimensional shape around a corner using ultrafast time-of-flight imaging. Nature communications, 3:745, 2012. 
[38] A. Velten, D. Wu, A. Jarabo, B. Masia, C. Barsi, C. Joshi, E. Lawson, M. Bawendi, D. Gutierrez, and R. Raskar. Femto-photography: capturing and visualizing the propagation of light. ACM Transactions on Graphics (TOG), 32(4):44, 2013.

[39] F. Villa, D. Bronzi, Y. Zou, C. Scarcella, G. Boso, S. Tisa, A. Tosi, F. Zappa, D. Durini, S. Weyers, et al. Cmos spads with up to $500 \mu \mathrm{m}$ diameter and 55\% detection efficiency at $420 \mathrm{~nm}$. Journal of Modern Optics, 61(2):102-115, 2014.

[40] Z. Zhang. Iterative point matching for registration of free-form curves and surfaces. International journal of computer vision, 13(2):119-152, 1994.

[41] Z. Zhou, H. Jin, and Y. Ma. Robust plane-based structure from motion. In Computer Vision and Pattern Recognition (CVPR), 2012 IEEE Conference on, pages 1482-1489. IEEE, 2012. 